\title{
In-vitro function of upstream visfatin polymorphisms that are associated with adverse cardiometabolic parameters in obese children
}

Delicia Shu Qin Ooi ${ }^{1,2+}$, Siong Gim Ong ${ }^{1,2+}$, Chew Kiat Heng ${ }^{1,2}$, Kah Yin Loke $e^{1,2}$ and Yung Seng Lee $e^{1,2,3,4^{*}}$

\begin{abstract}
Background: Visfatin is an adipokine associated with glucose and lipid metabolism. We previously reported two visfatin upstream single nucleotide polymorphisms (SNPs), c.-3187G > A (rs11977021) and c.-1537C > T (rs61330082), which were in perfect linkage disequilibrium, in a Singaporean cohort of severely obese children and are associated with visfatin level and adverse cardiometabolic parameters. We aim to functionally characterize the effect of c.$3187 \mathrm{G}>\mathrm{A}$ and c.-1537C > T SNPs on basal transcriptional activity.

Methods: A $1.6 \mathrm{~kb}$ and $3.7 \mathrm{~kb}$ upstream promoter region of the visfatin gene was amplified by polymerase chain reaction and separately cloned into luciferase reporter vectors. Successful clones were transfected into human embryonic kidney (HEK293T) and human breast carcinoma (MCF7) cells and in-vitro dual-luciferase assay was performed. Electrophoretic mobility shift assay (EMSA) was also conducted to examine the binding affinity between transcription factors and visfatin promoter sequences.

Results: Variant promoter with only c.-1537C > T SNP did not show a change in transcriptional activity as compared to the wild type. However, variant promoter with both c.-3187G > A and c.-1537C > T SNPs showed a statistically significant increase of 1.41 fold $(p<0.01)$ in transcriptional activity. The longer 3.7kbp visfatin promoter sequence was also shown to have significantly higher transcriptional activity $(p<0.05)$ as compared to the shorter $1.6 \mathrm{kbp}$ visfatin promoter. Both c.3187G > A and c.-1537C > T variants showed an increased binding with nuclear protein.

Discussion and conclusions: We have demonstrated for the first time that visfatin variant promoter with both c.3187G > A and c.-1537C > T SNPs result in an increase in transcriptional activity. This supports our previous finding and postulation that these SNPs contribute to elevated visfatin levels which may mediate higher triglyceride levels, severe systolic blood pressure and severe hypertension in obese children. These SNPs may co-operatively affect enhancer or silencer function to regulate transcriptional activity. In conclusion, this study shows that upstream visfatin SNPs could potentially affect phenotypic outcome in obese children through alteration of circulating visfatin level.
\end{abstract}

Keywords: Childhood obesity, Visfatin promoter, Luciferase assay, Polymorphisms, Variants

\footnotetext{
*Correspondence: paeleeys@nus.edu.sg

${ }^{\dagger}$ Equal contributors

${ }^{1}$ Department of Paediatrics, Yong Loo Lin School of Medicine, National

University of Singapore, Singapore, Singapore

${ }^{2}$ Division of Paediatric Endocrinology and Diabetes, Khoo Teck Puat-National

University Children's Medical Institute, National University Health System,

Singapore, Singapore

Full list of author information is available at the end of the article
}

\section{Biomed Central}

(c) The Author(s). 2016 Open Access This article is distributed under the terms of the Creative Commons Attribution 4.0 International License (http://creativecommons.org/licenses/by/4.0/), which permits unrestricted use, distribution, and reproduction in any medium, provided you give appropriate credit to the original author(s) and the source, provide a link to the Creative Commons license, and indicate if changes were made. The Creative Commons Public Domain Dedication waiver (http://creativecommons.org/publicdomain/zero/1.0/) applies to the data made available in this article, unless otherwise stated. 


\section{Background}

Visfatin is an adipokine secreted by adipose tissues [1] and plasma visfatin was shown to correlate with obesity and inflammation [2, 3]. The human visfatin gene (NC_000007.13) is mapped at 7q22.3 on the long arm of chromosome 7, it spans at an approximate length of $34.7 \mathrm{kbp}$ and consists of 11 exons and 10 introns [4]. The upstream promoter sequence of visfatin contains multiple binding sites for transcription factors such as nuclear factor-1 (NF-1), activator protein (AP-1 and AP-2), specificity protein-1 (SP-1) and glucocorticoid receptor (GR) [4]. Studies have identified several common upstream single nucleotide polymorphisms (SNPs) found to be associated with inflammation and parameters of glucose and lipid metabolism [5-8]. Among the reported upstream SNPs, the c.-1537C > T SNP (rs61330082) has been shown to cause a reduction in the transcriptional activity of luciferase reporter assay [9, 10]. However, another study by Tokunaga et al. found no difference in reporter gene expression between $\mathrm{C}$ and $\mathrm{T}$ allele of c.-1537C > T SNP [11]. Our group has previously reported perfect linkage disequilibrium between rs61330082 and another SNP, c.-3187C > T (rs11977021) which was found to be significantly associated with total plasma cholesterol and LDLcholesterol levels in French-Canadian subjects [5]. These two SNPs were also shown to be associated with obesity, severe systolic blood pressure, severe hypertension, plasma visfatin and triglyceride levels in our cohort of local severely obese children [12]. We hypothesized that the two upstream promoter SNPs (rs61330082 and rs11977021) may have a functional effect on the transcriptional activity of the visfatin gene.

\section{Methods}

\section{PCR, cloning and construction of visfatin plasmid}

Polymerase chain reaction (PCR) was performed to amplify the upstream promoter region of visfatin. The DNA samples of unrelated obese subjects identified to be homozygous for the respective wild-type and variant alleles of the 2 SNPs (rs61330082 and rs11977021) at perfect linkage disequilibrium were used for PCR [12]. The primers used to amplify an amplicon of $3.7 \mathrm{kbp}$ upstream from start codon ATG of visfatin gene were 5'-GTA CTC GAG GCC GGT TAG GAG AGT GCA GCA CAG-3' (forward) and 5'- GAA CTA AGA TCT CTC GGG CCG GAG GAC AGG GGC -3'(reverse). The primers used to amplify an amplicon of $1.6 \mathrm{kbp}$ upstream were 5'-GAC CTC GAG TGT TTC AAA CCT CGT TGC T-3' (forward) and 5'GAA CTA AGA TCT CTC GGG CCG GAG GAC AGG GGC -3'(reverse). The visfatin insert was then cloned into pGL4.10 luciferase reporter vector [Promega, USA] through BglII and XhoI restriction enzyme digestion. Successful clones were obtained and random variants in the clones were rectified by site-directed mutagenesis. The sequences of all plasmids were verified by DNA sequencing to ensure no other mutations were present (Additional files 1 and 2: Figures S1 and S2).

\section{Cell transfection and luciferase assays}

Human embryonic kidney cells, HEK293 and human breast cancer cells (MCF-7) were grown to $90 \%$ confluency. The seeding density of the cells was $\sim 5 \times 10^{4}$ cells/well and they were pre-cultured in 24-well tissue culture plate for $24 \mathrm{~h}$.

Cells were co-transfected with $1 \mathrm{ng} / \mu \mathrm{l}$ of visfatin pGL4.10 luciferase plasmid and $0.025 \mathrm{ng} / \mu \mathrm{l}$ of Renilla luciferase reporter vector using $0.03 \mu \mathrm{l} / \mu \mathrm{l}$ of Lipofectamine 2000 [Invitrogen, USA]. The transfected cells were incubated at $37{ }^{\circ} \mathrm{C}$ with $5 \% \mathrm{CO}_{2}$ overnight for $24 \mathrm{~h}$ before they were used for luciferase assay study. The functionality of the visfatin insert was determined by dualluciferase reporter assay [Promega, USA] according to manufacturer's instructions. The assay for each visfatin insert was performed in triplicates and the experiment was performed thrice. All the 3 transfection experiments showed similar fold-change. However, due to the variable transfection efficiency, we presented the transfection results as the mean and $\mathrm{SD}$ of the triplicates in one experiment setting. The results for other 2 biological replicates are shown in Additional file 3: Figure S3.

\section{Prediction of the functional consequences of the variants}

We investigated whether the promoter variants have any effect on putative transcription factor binding sites within the visfatin gene using PROMO. The parameters used in ALGGEN-PROMO program were "only human factors" and "only human sites". (http://alggen.lsi.upc.es/cgibin/promo_v3/promo/promoinit.cgi?dirDB=TF_8.3, last accessed on $30^{\text {th }}$ May 2014) $[13,14]$. MotifMap was also used to check the nucleotide sequence of the transcription factor binding sites.

\section{Electrophoretic mobility shift assay (EMSA)}

We extracted nuclear protein from cultured MCF7 cells using the NE-PER nuclear and cytoplasmic extraction kit according to manufacturer's instructions [ThermoFisher Scientific, USA]. Biotin-labeled probes of the visfatin promoter sequence containing the respective c.-3187G $>\mathrm{A}$ and c.- $1537 \mathrm{C}>\mathrm{T}$ variant were synthesized [Integrated DNA technologies, USA]. The probes represent a short section (11 bases) of the visfatin promoter sequence and were designed to include 5 bases before and after the respective SNP. The probe sequences are c.-3187G: 5'-ACTGAGGTCAA-3'; c.-3187A: 5'-ACTGAAGTCAA-3'; c.-1537C: 5'AGTGCCTGGTG-3'; C.-1537 T: 5'- AGTGCTTGGTG-3'. The biotin-labeled probes were incubated with or without the nuclear protein extract and in the presence or absence of unlabeled probes. EMSA was carried out using the LightShift chemiluminescent EMSA kit according to 
manufacturer's instructions [ThermoFisher Scientific, USA]. The experiment was performed twice (Additional file 4: Figure S4) and the density of each band was quantified by GeneTool [Syngene, Cambridge, England].

\section{Statistical analyses}

Data are expressed as mean \pm standard deviation (SD). The difference in luciferase activity between different visfatin promoter sequences was analyzed by the Student's $t$ test. Paired sample $t$-test was used to analyze the difference in EMSA band intensity between wild-type and variant promoter sequence [SPSS Version 22, USA].

\section{Results}

Transcription factor binding sites in visfatin promoter sequence

We used the transcription factor binding site prediction program PROMO to predict any alteration in transcription factor binding affinity in visfatin promoter sequence previously found to contain single nucleotide polymorphisms (SNPs), rs61330082 (c.-3187G > A) and rs11977021 (c.$1537 \mathrm{C}>\mathrm{T})$. PROMO predicted the alteration of the transcription factor estrogen receptor-alpha (ER-alpha) binding site by c.-3187G $>$ A variant while c. $-1537 \mathrm{C}>\mathrm{T}$ variant was predicted to alter activating protein-2alphaA (AP-2alphaA) transcription factor binding site as shown in Fig. 1.

\section{Comparison of transcriptional activity between visfatin promoter variants}

The basal transcriptional activity was measured as the invitro luciferase activity of the different visfatin promoter sequences in transiently transfected MCF7 and HEK293T cells as shown in Fig. 2. The transcriptional activity of the variant $3.7 \mathrm{kbp}$ promoter sequence containing both c.3187G > A and c.-1537C > T SNPs was 1.27 and 1.41 fold higher than that of wild-type $3.7 \mathrm{kbp}$ promoter in MCF7 and HEK293T cells respectively. The variant $1.6 \mathrm{kbp}$ visfatin promoter sequence containing only c. $-1537 \mathrm{C}>\mathrm{T}$ SNP showed no significant change in luciferase activity as compared to wild-type 1.6kbp promoter in both MCF7 and HEK293T cells. The transcriptional activity of the wild-type $3.7 \mathrm{kbp}$ promoter sequence was 1.08 and 1.13 fold $(p=$ 0.014 ) higher than that of wild-type $1.6 \mathrm{kbp}$ promoter in MCF7 and HEK293T cells respectively. The transcriptional activity of the variant $3.7 \mathrm{kbp}$ promoter sequence was 1.27 and 1.48 fold higher than that of variant $1.6 \mathrm{kbp}$ promoter in MCF7 and HEK293T cells respectively. The luciferase assay was performed in triplicates $(n=3)$ for each promoter sequence and a minimal amount of luciferase activity $(0.21$ \pm 0.03 and $0.27 \pm 0.02$ ) was detected in HEK293T and MCF cells transfected with control pGL4.10 luciferase plasmid.

\section{Binding of transcription factors to visfatin variant promoter sequence}

EMSA data showed that the variant alleles in the respective c.-3187G > A and c.-1537C > T SNPs have a significant increased binding with nuclear protein (Fig. 3a, b). The binding between the visfatin variant promoter sequence and nuclear protein is assessed by densitometry and the large standard deviation values were due to comparison between 2 separate EMSA experiments (Fig. 3c).

\section{Discussion and conclusion}

In this study, we functionally characterized two upstream visfatin promoter SNPs, c.-3187G > A (rs11977021) and c.$1537 \mathrm{C}>\mathrm{T}$ (rs61330082), that were previously detected in the upstream promoter region of visfatin gene in our cohort of severely obese children [12]. Through the use of the transcription factor binding site prediction program PROMO, the c.-3187G > A and c.-1537C > T SNPs in the visfatin promoter were predicted to alter the ER-alpha and AP-2alphaA transcription factor binding sites respectively (Fig. 1). Wang et al. reported that the c. $1537 \mathrm{C}>\mathrm{T}$ SNP altered promoter binding to AP-1 [9] but the study did not examine the promoter binding affinity to AP-2alpha2 which can also be a likely transcription factor that is affected by the c. $-1537 \mathrm{C}$ > $\mathrm{T}$ SNP due to the homology between the promoter sequence and AP-2alphaA as predicted by PROMO.

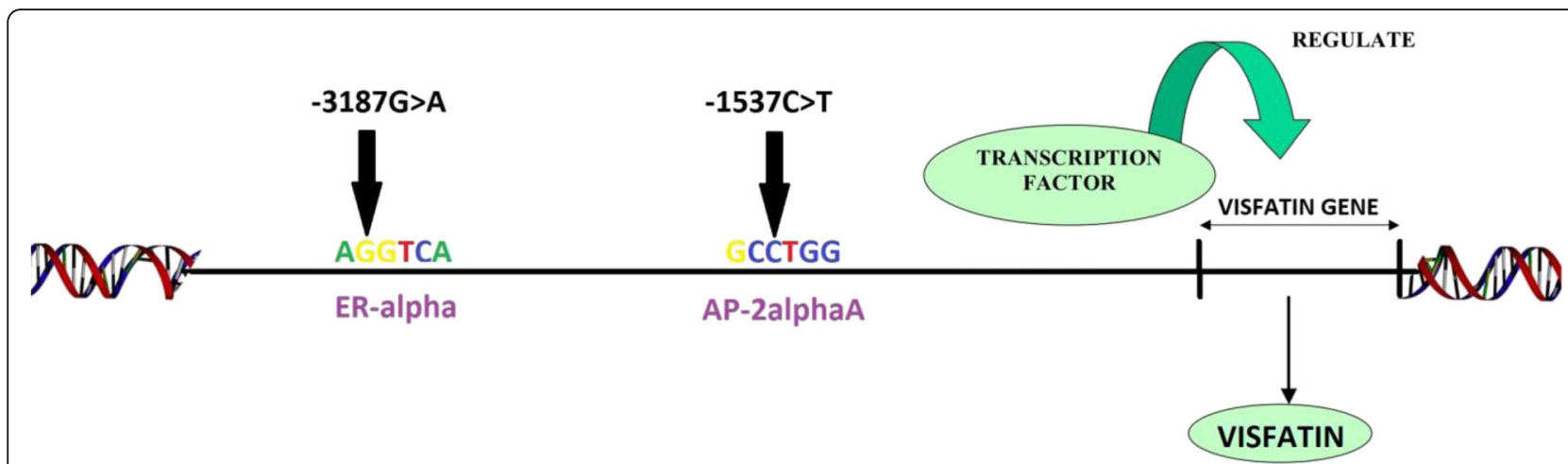

Fig. 1 Promoter region of visfatin gene. The sequence representing the ER-alpha and AP-2alphaA transcription factor binding sites located respectively at -3187 and -1537 of the visfatin promoter region as predicted by PROMO are shown 

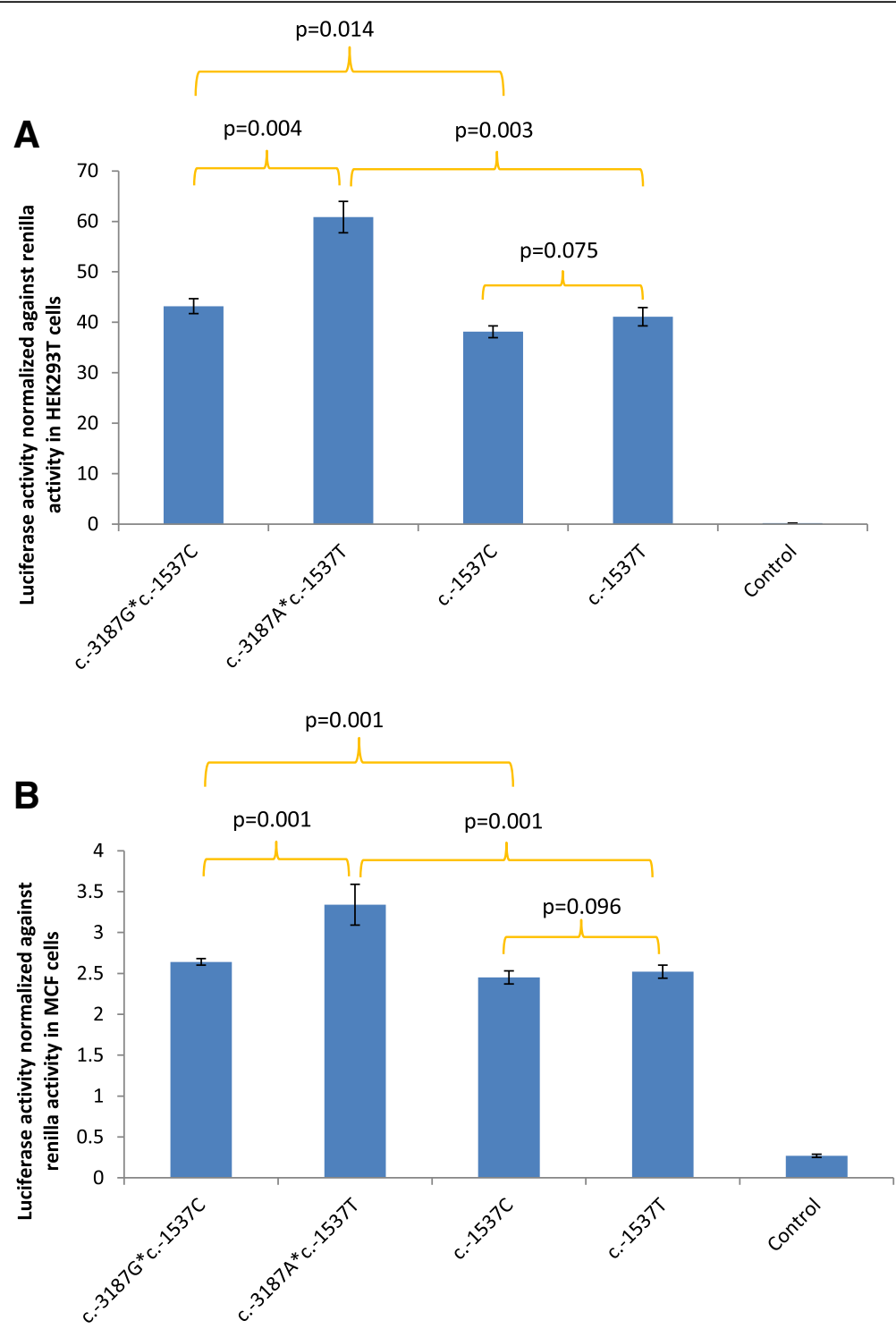

Fig. 2 Basal transcriptional activity of visfatin promoter variants as measured in-vitro by luciferase assay. The average relative transcriptional activities of the visfatin promoter variants in a HEK293T and b MCF7 cells are indicated in shaded bars and the standard deviation (SD) is represented by the error bars. The $p$-values for the comparison of in-vitro transcriptional activity between the promoter sequences are stated. c.-3187* c. -1537 indicates a $3.7 \mathrm{kbp}$ promoter sequence containing both c.-3187 and c.-1537 SNPs, while c.-1537 indicates a $1.6 \mathrm{kbp}$ promoter sequence containing only c.-1537 SNP

The $3.7 \mathrm{kbp}$ visfatin variant promoter sequence containing both c.-3187G > A and c.-1537C > T SNPs was shown to have a significantly higher luciferase activity than that of the wild-type 3.7kbp promoter (Fig. 2). This indicated that the 3.7kbp variant promoter has an increased transcriptional activity and this is in accordance with our previous finding that the two SNPs were associated with elevated serum visfatin $(6.17 \pm 0.76 \mathrm{ng} / \mathrm{ml}$ vs. $3.92 \pm$ $0.44 \mathrm{ng} / \mathrm{ml}$ ) in our cohort of severely obese children [12].

In addition, we examined the transcriptional activity of the $1.6 \mathrm{kbp}$ variant visfatin promoter containing only c.$1537 \mathrm{C}>\mathrm{T}$ SNP and found that there is no significant difference in transcriptional activity as compared to wild-type 1.6kbp promoter (Fig. 2). Our finding is similar to that reported by Tokunaga et al. who also found no difference in transcriptional activity between variant 1.6kbp visfatin promoter containing c.-1537C $>\mathrm{T}$ SNP and wild-type $1.6 \mathrm{kbp}$ promoter [11]. However, this differs from the reports by Wang et al. and Ye et al. who showed that c.-1537C > T SNP was associated with lower transcriptional activity $[9,10]$. This difference may be attributed to the length of promoter sequence included in the luciferase reporter assay. While Wang et al. and Ye et al. have included $\sim 216 \mathrm{bp}$ and $\sim 147 \mathrm{bp}$ of the visfatin promoter sequence respectively $[9,10]$, our study has included a $\sim 1.6 \mathrm{kbp}$ promoter sequence upstream of the 

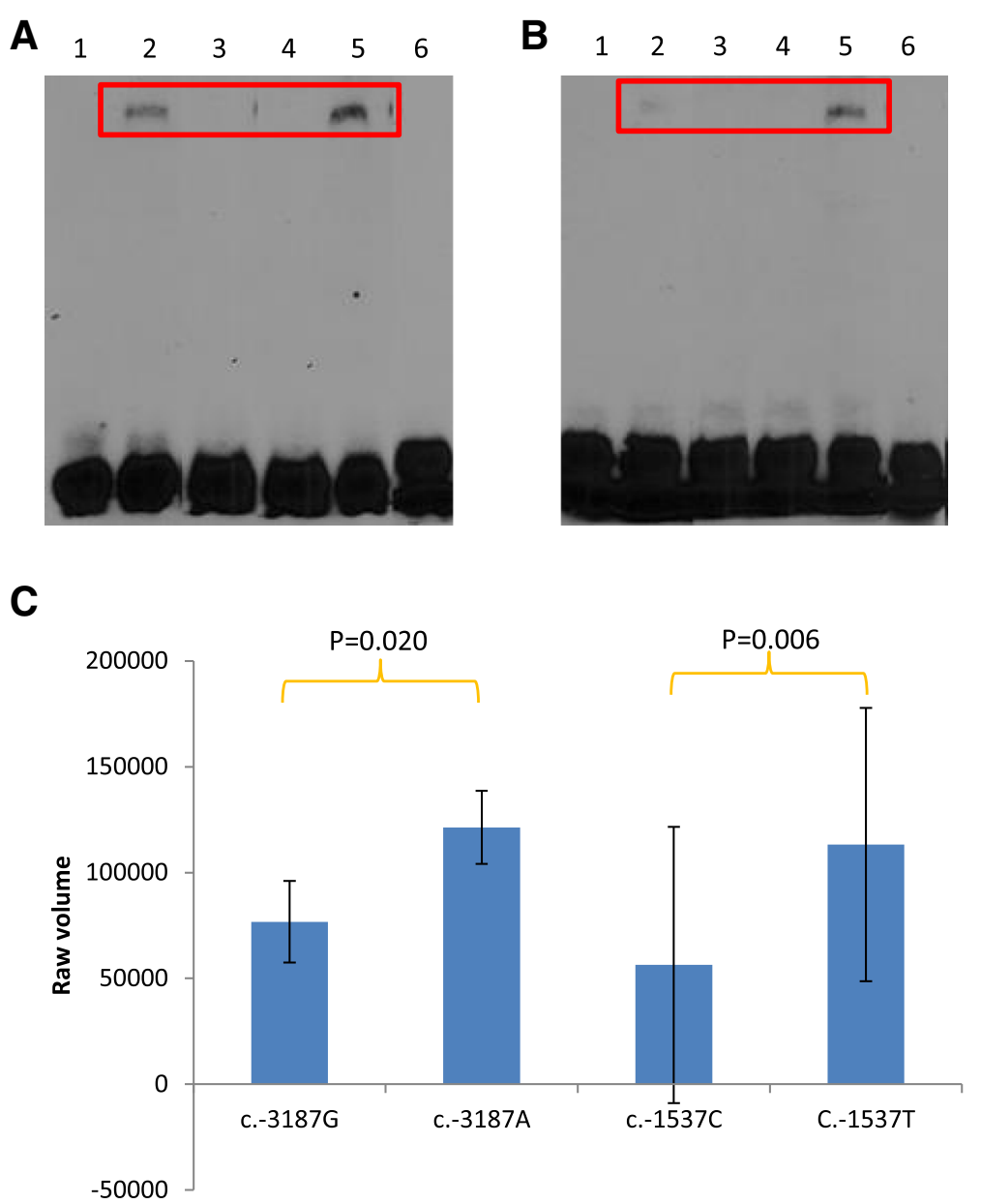

Fig. 3 Binding of transcription factors to visfatin promoter variants as measured in-vitro by EMSA. a EMSA for c.-3187G > A SNP only, b EMSA for c.-1537C > T SNP only, c Assessment of binding between visfatin SNPs and nuclear protein, the EMSA band density are indicated in shaded bars and the standard deviation (SD) between 2 EMSA experiments is represented by the error bars. (1): Wild-type sequence without nuclear protein, (2): Wild-type sequence with nuclear protein, (3): Wild-type sequence with nuclear protein and unlabeled probe, (4): Variant sequence without nuclear protein, (5): Variant sequence with nuclear protein, (6): Variant sequence with nuclear protein and unlabeled probe

ATG start codon similar to that in the paper by Tokunaga et al. [11]. The putative upstream promoter region of visfatin gene has been shown to contain multiple transcription factor binding sites [4]. Hence, a full promoter sequence from the ATG start site may account for the effect of other transcription factors on the transcriptional activity of the promoter.

The longer $3.7 \mathrm{kbp}$ wild-type visfatin promoter was found to have a significantly higher transcriptional activity as compared to the shorter $1.6 \mathrm{kbp}$ wild-type promoter. Moreover, the longer $3.7 \mathrm{kbp}$ variant promoter was also found to exhibit a higher transcriptional activity as compared to the shorter $1.6 \mathrm{kbp}$ variant promoter (Fig. 2). Though the in-vivo significance of this finding is unclear, we speculate that it might imply that the longer $3.7 \mathrm{kbp}$ region is more representative of the putative promoter. The higher transcriptional activity of the wild-type $3.7 \mathrm{kbp}$ promoter may also contribute in part to the accentuated in-vitro activity of $3.7 \mathrm{kbp}$ variant promoter. In addition, the two SNPs, c.-3187G > A and c.-1537C > $\mathrm{T}$, at perfect linkage disequilibrium may have combinatorial effect on the transcriptional activity of the promoter as it has been shown that variants at linkage disequilibrium located at multiple enhancer sites may cooperatively dictate transcript expression [15]. Therefore, unlike previous studies which only examine the effect of c.-1537C $>\mathrm{T}$ SNP, our study takes into account distal promoter variants that may have combinatorial effect on the transcriptional activity of the promoter.

Both c.-3187G > A and c.-1537C > T SNPs have been reported as expression quantitative trait loci (eQTLs) in left ventricular heart tissue with trends of decreasing expression as the genotype changes from homozygous wild-type to heterozygous to homozygous variant [16]. 
However, our results are contrary to that of the reported eQTLs and this may be possibly due to these SNPs/reported eQTLs having different effects on different cell types, resulting in the difference in expression level. Hence, the choice of cell model should be taken into consideration when examining the effect of SNPs on expression level.

Data from our EMSA experiments indicated an increased binding of nuclear protein to the variant $\mathrm{A}$ and $\mathrm{T}$ alleles of c.-3187G > A and c.-1537C > T SNPs respectively (Fig. 3). This supports the increased transcriptional activity observed in the $3.7 \mathrm{kbp}$ visfatin variant promoter sequence (Fig. 2). Although c.-1537C > T SNP also showed an increase in nuclear protein binding, it did not result in an increase in transcriptional activity. This may be due to other upstream SNPs at different regions of the promoter sequence which may cooperatively affect enhancer or silencer function to regulate transcriptional activity.

Since c.-3187G > A does not exist in solitary without c.$1537 \mathrm{C}>\mathrm{T}$ in our obese children cohort, we did not examine the effect of c.-3187G > A SNP alone on the transcriptional activity. Hence, we could not determine if both SNPs are needed to work cooperatively in order to exert an effect on transcriptional activity. In addition, we did not examine the specific transcription factor that is affected by the c. $-3187 \mathrm{G}>\mathrm{A}$ and c. $-1537 \mathrm{C}>\mathrm{T}$ SNPs respectively. Hence, we are not able to determine if the SNPs have affected binding with ER-alpha and AP-2alphaA transcription factors as predicted by PROMO.

In conclusion, we have demonstrated for the first time that visfatin variant promoter with both c.-3187G $>$ A and c.-1537C $>$ T SNPs result in increased in-vitro transcriptional activity. This supports our previous finding and postulation that these SNPs contribute to elevated visfatin levels which mediate higher triglyceride levels, severe systolic blood pressure and severe hypertension in obese children. This study supports the role of these upstream visfatin SNPs which could potentially affect phenotypic outcome in obese children through alteration of circulating visfatin level.

\section{Additional files}

Additional file 1: Supplementary Figure 1. Plasmid sequence for wildtype visfatin promoter. (DOCX $867 \mathrm{~kb}$ )

Additional file 2: Supplementary Figure 2. Plasmid sequence for variant visfatin promoter. (DOCX $1313 \mathrm{~kb}$ )

Additional file 3: Supplementary Figure 3. Biological replicates for luciferase assays. (DOCX $233 \mathrm{~kb}$ )

Additional file 4: Biological replicate for EMSA. (DOCX $372 \mathrm{~kb}$ )

\section{Abbreviations}

AP-1/AP-2: Activator protein-1/2; AP-2alphaA: Activating protein-2alphaA; EMSA: Electrophoretic Mobility Shift Assay; eQTL: Expression quantitative trait loci; ER-alpha: Estrogen receptor-alpha; GR: Glucocorticoid receptor;
HEK: Human embryonic kidney; NF-1: Nuclear factor-1; PCR: Polymerase chain reaction; SD: Standard deviation; SNP: Single nucleotide polymorphism; SP-1: Specificity protein-1

\section{Acknowledgements}

We would like to extend our gratitude to Dr Ratha, Dr Juwita and Miss Rachel Tham for allowing us to conduct our experiments using their lab facility.

\section{Funding}

The work was supported by the Singapore Paediatric Society Research Grant 2013 and Singapore Institute for Clinical Sciences (SICS) core funding of Lee Yung Seng (C8019).

\section{Availability of data and materials}

All data supporting the findings in this study can be found within the manuscript and supplementary figures (Additional files 1, 2, 3 and 4: Figures S1, S2, S3, S4).

\section{Authors' contributions}

DO and SG carried out the functional characterization studies, participated in bioinformatics analysis and drafted the manuscript. CK was involved in the revision of the manuscript and provided technical advices and support for the molecular experiments. KY participated in the collection of genetic material from patients and provided guidance in the drafting of the manuscript. YS provided technical advices and support for the molecular experiments, participated in the drafting and revision of manuscript. All authors read and approved the final manuscript.

\section{Competing interests}

The authors declared that no competing interests exist.

\section{Consent for publication}

Not applicable.

\section{Ethics approval and consent to participate}

This study was approved by the National Healthcare Group Domain Specific Review Board, Singapore (DSRB Ref C/00/501) and informed consent was obtained from subjects and parents.

\section{Author details}

'Department of Paediatrics, Yong Loo Lin School of Medicine, National University of Singapore, Singapore, Singapore. ${ }^{2}$ Division of Paediatric Endocrinology and Diabetes, Khoo Teck Puat-National University Children's Medical Institute, National University Health System, Singapore, Singapore. ${ }^{3}$ Singapore Institute for Clinical Sciences, Agency of Science, Technology and Research, Singapore, Singapore. ${ }^{4}$ Department of Paediatrics, Yong Loo Lin School of Medicine, NUHS tower block, Level 12, 1E Kent Ridge Road, Singapore 119228, Singapore.

Received: 9 May 2015 Accepted: 18 November 2016

Published online: 25 November 2016

\section{References}

1. Berndt J, Kloting N, Kralisch S, Kovacs P, Fasshauer M, Schon MR, Stumvoll M, Bluher M. Plasma visfatin concentrations and fat depot-specific mRNA expression in humans. Diabetes. 2005;54(10):2911-6.

2. Unluturk U, Harmanci A, Yildiz BO, Bayraktar M. Dynamics of nampt/visfatin and high molecular weight adiponectin in response to oral glucose load in obese and lean women. Clin endocrinol (Oxf). 2010:72(4):469-74.

3. Brentano F, Schorr O, Ospelt C, Stanczyk J, Gay RE, Gay S, Kyburz D. Pre-B cell colony-enhancing factor/visfatin, a new marker of inflammation in rheumatoid arthritis with proinflammatory and matrix-degrading activities. Arthritis rheum. 2007:56(9):2829-39.

4. Ognjanovic S, Bao S, Yamamoto SY, Garibay-Tupas J, Samal B, BryantGreenwood GD. Genomic organization of the gene coding for human preB-cell colony enhancing factor and expression in human fetal membranes. J mol endocrinol. 2001;26(2):107-17.

5. Bailey SD, Loredo-Osti JC, Lepage P, Faith J, Fontaine J, Desbiens KM, Hudson TJ, Bouchard C, Gaudet D, Perusse L, et al. Common polymorphisms in the promoter of the visfatin gene (PBEF1) influence plasma insulin levels in a French-Canadian population. Diabetes. 2006:55(10):2896-902. 
6. Bottcher Y, Teupser D, Enigk B, Berndt J, Kloting N, Schon MR, Thiery J Bluher M, Stumvoll M, Kovacs P. Genetic variation in the visfatin gene (PBEF1) and its relation to glucose metabolism and fat-depot-specific messenger ribonucleic acid expression in humans. J clin endocrinol metab. 2006;91(7):2725-31.

7. Yan JJ, Tang NP, Tang JJ, Jia EZ, Wang MW, Wang QM, Zhu J, Yang ZJ, Wang LS, Huang J. Genetic variant in visfatin gene promoter is associated with decreased risk of coronary artery disease in a Chinese population. Clin chim acta; int j clin chem. 2010;411(1-2):26-30.

8. Zhang YY, Gottardo L, Thompson R, Powers C, Nolan D, Duffy J, Marescotti MC, Avogaro A, Doria A. A visfatin promoter polymorphism is associated with lowgrade inflammation and type 2 diabetes. Obesity. 2006;14(12):2119-26.

9. Wang YS, Gao W, Li HF, Wang ZM, Zhu J, Zhao H, Yan JJ, Jia EZ, Yang ZJ, Wang LS. Mechanistic insights into the link between visfatin gene C-1535 T polymorphism and coronary artery disease: an in vitro study. Mol cell biochem. 2012;363(1-2):315-22.

10. Ye SQ, Simon BA, Maloney JP, Zambelli-Weiner A, Gao L, Grant A, Easley RB, McVerry BJ, Tuder RM, Standiford T, et al. Pre-B-cell colony-enhancing factor as a potential novel biomarker in acute lung injury. Am $\mathrm{j}$ respir crit care med. 2005;171(4):361-70.

11. Tokunaga A, Miura A, Okauchi Y, Segawa K, Fukuhara A, Okita K, Takahashi M, Funahashi T, Miyagawa J, Shimomura I, et al. The -1535 promoter variant of the visfatin gene is associated with serum triglyceride and HDLcholesterol levels in Japanese subjects. Endocr j. 2008;55(1):205-12.

12. Ooi SQ, Chan RM, Poh LK, Loke KY, Heng CK, Chan YH, Gan SU, Lee KO, Lee YS. Visfatin and its genetic variants are associated with obesity-related morbidities and cardiometabolic risk in severely obese children. Pediatr obes. 2014;9(2):81-91.

13. Messeguer X, Escudero R, Farre D, Nunez O, Martinez J, Alba MM. PROMO: detection of known transcription regulatory elements using species-tailored searches. Bioinformatics. 2002;18(2):333-4.

14. Farre D, Roset R, Huerta M, Adsuara JE, Rosello L, Alba MM, Messeguer X. Identification of patterns in biological sequences at the ALGGEN server: PROMO and MALGEN. Nucleic acids res. 2003;31(13):3651-3.

15. Corradin O, Saiakhova A, Akhtar-Zaidi B, Myeroff L, Willis J, Cowper-Sal lari R, Lupien M, Markowitz S, Scacheri PC. Combinatorial effects of multiple enhancer variants in linkage disequilibrium dictate levels of gene expression to confer susceptibility to common traits. Genome res. 2014;24(1):1-13.

16. http://www.gtexportal.org/home/eqtls/tissue?tissueName=All, last Accessed on 14th Sept 2016.

\section{Submit your next manuscript to BioMed Central and we will help you at every step:}

- We accept pre-submission inquiries

- Our selector tool helps you to find the most relevant journal

- We provide round the clock customer support

- Convenient online submission

- Thorough peer review

- Inclusion in PubMed and all major indexing services

- Maximum visibility for your research

Submit your manuscript at www.biomedcentral.com/submit

) Biomed Central 